\title{
Marker-and-cell and Chorin finite difference modeling for fluid flow in a single fracture*
}

\author{
Duoxing Yang * \\ Institute of Crustal Dynamics, China Earthquake Administration, Beijing 100085, China
}

\begin{abstract}
It is important to set up a detailed dynamic model of the fluid flow through fractures for understanding many fluid processes in Earth sciences. Numerical simulation is a popular tool for exploring these processes. The objective of this study is to understand fluid flow in fractures. Contrary to the conventional macro-scale modeling approach, micro-scale simulation is carried out. The Navior-Stokes equation solver was developed by a staggered marker-and-cell and the Chorin pressure iterating finite difference approach. We analyze the effects of the Reynolds number and the frequency of pressure fluctuations on flow mainly through visualization. A significant result is that the effect of pressure fluctuation-induced fluid flow can be observed in a broader frequency range. The peak velocity shifts along the spatial axis depending upon the frequency of the pressure fluctuation. An effective frequency band of the pressure fluctuation was identified which dominates dynamic behavior of the flow. Another major finding is that there exits a critical frequency of the pressure fluctuation which controls approximately the flow dynamic behavior. We conclude that it is only possible to estimate the flow behavior from pressure fluctuation, if effective frequency range is properly accounted for.
\end{abstract}

Key words: marker-and-cell; frequency; pressure fluctuation; Reynolds number CLC number: P315.72 3 Document code: A

\section{Introduction}

Fluid flow through fractures is important in environmental and petroleum engineering and geological sciences (Cardenas et al, 2007; Luis et al, 2007). Problems addressed include groundwater flow, contaminant transport, nuclear waste management, and enhanced hydrocarbon recovery ( $\mathrm{Li}$ et al, 2005). Hence, understanding the water flow behavior in a single fracture is essential for investigating groundwater flow and solute transport in fractured rock formations in the context of water supply, remediation of dissolved contaminant or projecting long-term nuclear waste repositories. Karpyn et al (2007) studied the effects of fracture morphology on the distribution and transport of immiscible fluid phases through a vertical fracture by using micro-computed tomography (MCT). Numerical modeling is used as a tool for studying the geometrical and hydraulic properties of a single fracture (Cardenas et al, 2007; Steven et al, 1986). The mathematical representation of fluid dynamics in a single fracture is generally simplified because of

\footnotetext{
* Received 20 April 2009; accepted in revised form 1 September 2009; published 10 October 2009 .

• Corresponding author. e-mail: yangdx@mail.igcas.ac.cn
}

observational and computational limitations as well as scaling issues (Cardenas et al, 2007). Fluid flow in a single fracture is also simulated using the Reynolds equation (Brown et al, 1995; Giacomini et al, 2008). Recent studies suggest that this depth-averaged approach underestimates head loss in regions with changing aperture (Michael and Nicholl, 2001), and the Reynolds equation tends to give higher flow rates (Giacomini et al, 2008). Advancing our fundamental understanding of fluid in discrete fractures requires staying within the framework of the non-simplified NavierStokes equations (NSE) (Brown, 1995; Zimmerman et al, 2004; Cardenas et al, 2007). Several researchers have solved the NSE for single fracture following different formulations and different numerical methods. Brown et al (1995) used cellular automata models that retrieve the NSE at continuum scale, while Brush and Thomson (2003) and Cardenas et al (2007) directly solved the NSE. The simulations by Brown et al (1995) and Brush and Thomson (2003) considered idealized (i.e., sinusoidal) or statistically generated synthetic fractures. On the contrary, Cardenas et al (2007) used a real undisturbed fracture geometry for both surfaces and inverted the output using analytical solutions to the one dimensional advection-dispersion equation to retrieve effective dis- 
persion coefficients and found slight variations in fracture characteristics, resulting in significantly different flow and transport behavior. Many studies focus on the effects of fracture morphology and distribution of surface roughness on the distribution and transport of fluid phases through a single fracture (Waite et al, 1998; Kim et al, 2003; Cardenas et al, 2007), but neither simultaneously consider effects of the Reynolds number and the frequency of pressure fluctuations on fluid flow through a single fracture in their work.

The thrust of our study is to develop a numerical model based on the basic physics of fluid flow in the earth, and to implement it to simulate fracture flow and to analyze the effects of the Reynolds number and the frequency of pressure fluctuations on fluid flow mainly through numerical simulation. We conduct NSE-based flow simulations for single phase flow through a $2 \mathrm{D}$ idealized fracture. The numerical computations are performed using a finite difference method. Governing partial differential equations are transformed to algebraic ones by marker-and-cell (MAC) (Harlow, 1965) and Chorin pressure iteration method (Olivier and Grönig, 1986) over structured and staggered grids (Jaramillo et al, 2007). The MAC method involves a Lagrangian particle movement and an Eulerian flow field calculation (Harlow, 1965). The marker is used to track the centroid of the fluid element. Its velocity is obtained by averaging the Eulerian velocities in its vicinity.

\section{Governing equation and computa- tional method}

As the governing equations of fluid flow through a fracture, the continuity equation and the Navier-Stokes equations (NSE) for two-dimensional incompressible and viscous flow can be summarized as follows (Harlow, 1965):

$$
\nabla \cdot \boldsymbol{U}=0
$$

and

$$
\frac{\partial \boldsymbol{U}}{\partial t}+\boldsymbol{U} \cdot \nabla \boldsymbol{U}=-\nabla p+\frac{1}{R e} \nabla^{2} \boldsymbol{U},
$$

where $\boldsymbol{U}=[u, v]$ denote velocity vector, and $p, t$, and $R e$ are pressure, time, and Reynolds number, respectively. These parameters can be non-dimensionalized by $\boldsymbol{U}_{0}$ (reference velocity), $L$ (reference length), and $\mu$ (dynamic viscosity). The original governing equations are discretized by means of finite difference method.

\subsection{Discretization of equations}

To obtain a finite-difference expression for the NSE, we point out that for the spatial derivatives with respect to the respective space variables $x$ or $y$, the reference grid points are $(i+1 / 2, j)$ and $(i, j+1 / 2)$, respectively. Applying central discretization in space and the time derivative in equation (2) with a forward difference and staggered grids, we can obtain

$$
\begin{gathered}
\frac{u_{i+1 / 2, j}^{n+1}-u_{i+1 / 2, j}^{n}}{\Delta t}+\frac{1}{\Delta x}\left[\left(u_{i+1, j}^{n}\right)^{2}-\left(u_{i, j}^{n}\right)^{2}\right]+ \\
\frac{1}{\Delta y}\left[(u v)_{i+1 / 2, j+1 / 2}^{n}-(u v)_{i+1 / 2, j-1 / 2}^{n}\right]= \\
-\frac{1}{\Delta x}\left(p_{i+1, j}^{n+1}-p_{i, j}^{n+1}\right)+\frac{1}{R e}\left[\frac{u_{i+3 / 2, j}^{n}-2 u_{i+1 / 2, j}^{n}+u_{i-1 / 2, j}^{n}}{\Delta x^{2}}+\right. \\
\left.\frac{u_{i+1 / 2, j+1}^{n}-2 u_{i+1 / 2, j}^{n}+u_{i+1 / 2, j-1}^{n}}{\Delta y^{2}}\right]
\end{gathered}
$$

and

$$
\begin{gathered}
\frac{v_{i, j+1 / 2}^{n+1}-u_{i, j+1 / 2}^{n}}{\Delta t}+\frac{1}{\Delta y}\left[\left(v_{i, j+1}^{n}\right)^{2}-\left(v_{i, j}^{n}\right)^{2}\right]+ \\
\frac{1}{\Delta x}\left[(u v)_{i+1 / 2, j+1 / 2}^{n}-(u v)_{i-1 / 2, j+1 / 2}^{n}\right]= \\
-\frac{1}{\Delta y}\left(p_{i, j+1}^{n+1}-p_{i, j}^{n+1}\right)+\frac{1}{R e}\left[\frac{v_{i+1, j+1 / 2}^{n}-2 v_{i, j+1 / 2}^{n}+v_{i-1, j+1 / 2}^{n}}{\Delta x^{2}}+\right. \\
\left.\frac{v_{i, j+3 / 2}^{n}-2 v_{i, j+1 / 2}^{n}+v_{i, j-1 / 2}^{n}}{\Delta y^{2}}\right]
\end{gathered}
$$

Introduce the following equation:

$$
D_{i . j}^{n}=\frac{u_{i+1 / 2, j}^{n}-u_{i-1 / 2, j}^{n}}{\Delta x}+\frac{v_{i, j+1 / 2}^{n}-v_{i, j-1 / 2}^{n}}{\Delta y},
$$

then

$$
\begin{gathered}
\frac{p_{i+1, j}^{n+1}-2 p_{i, j}^{n+1}+p_{i-1, j}^{n+1}}{\Delta x^{2}}+\frac{p_{i, j+1}^{n+1}-2 p_{i, j}^{n+1}+p_{i, j+1}^{n+1}}{\Delta y^{2}}= \\
-\frac{D_{i, j}^{n+1}-D_{i, j}^{n}}{\Delta t}-\frac{\left(u_{i+1, j}^{n}\right)^{2}-2\left(u_{i, j}^{n}\right)^{2}+\left(u_{i-1, j}^{n}\right)^{2}}{\Delta x^{2}}- \\
\frac{\left(v_{i, j+1}^{n}\right)^{2}-2\left(v_{i, j}^{n}\right)^{2}+\left(v_{i, j-1}^{n}\right)^{2}}{\Delta y^{2}}-\frac{2}{\Delta x \Delta y} \cdot\left[(u v)_{i+1 / 2, j+1 / 2}^{n}+\right. \\
\frac{1}{\operatorname{Re}}\left[\frac{\left.(u v)_{i-1 / 2, j-1 / 2}^{n}-(u v)_{i+1 / 2, j-1 / 2}^{n}-(u v)_{i-1 / 2, j+1 / 2}^{n}\right]+}{\Delta x^{2}}-2 D_{i, j}^{n}+D_{i-1, j}^{n}\right. \\
\left.\frac{D_{i, j+1}^{n}-2 D_{i, j}^{n}+D_{i, j-1}^{n}}{\Delta y^{2}}\right],
\end{gathered}
$$

where the subscripts denote derivatives with respect to the respective space variables $x$ or $y$. Let us now exam- 
ine the properties of the MAC scheme for the computation of steady-state solutions. If the solution satisfies

$$
\frac{u_{i, j}^{n+1}-u_{i-1, j}^{n+1}}{\Delta x}+\frac{v_{i, j}^{n+1}-v_{i, j-1}^{n+1}}{\Delta y}<\varepsilon,
$$

the steady-state solutions can be obtained, where $\varepsilon$ is an accuracy dependent scale. The iterative process between velocity and pressure is performed from the Chorin pressure iterative approach as follows. Here, the calculation of $\left(D_{i, j}^{n+1}\right)^{m}$ from equation (5) is first obtained from the known flow field values, where $m$ is the iteration number. Hence, from equation (8),

$$
\left(p_{i, j}^{n+1}\right)^{m+1}=\left(p_{i, j}^{n+1}\right)^{m}-\lambda\left(D_{i, j}^{n+1}\right)^{m},
$$

a value of pressure $\left(p_{i, j}^{n+1}\right)^{m+1}$ is readily obtained. Now calculate velocities from the momentum equations (3) and (4). Repeating equation (5), we obtain the value of $D_{i, j}^{n+1}$. The above Chorin pressure-iteration approach is carried out for all grid points. The steps are repeated for a number of times until the steady state is achieved.

Repeat the above process for a large number of time steps. At each time step, the flow properties at all grid points will change from one time to the next. However, at large times, these changes become very small, and a steady-state is approached. This steady-state is the desired result, and the time-dependent technique is simply a means to that end.

\subsection{Stability criterion}

MAC is an explicit method, and, as most such methods, is not unconditionally stable. To guarantee stability, we have to make sure that these inequalities hold (Harlow, 1965)

$$
\lambda \leq \frac{\Delta x^{2} \Delta y^{2}}{\Delta t\left(\Delta x^{2}+\Delta y^{2}\right)} .
$$

Here, $\Delta t, \Delta x, \Delta y$ and $\lambda$ refer to the time step size, space step size and relaxation factor, respectively. $\lambda$ should be close to $0.9 \lambda_{\max }$ as much as possible.

\subsection{Boundary conditions and domain discretization}

Consider the two-dimensional incompressible and viscous flow through a single fracture sketched in Figure 1a. We assume that the fracture walls are no-slip boundaries while the inlets and outlets are prescribed pressures. In the outlet, we use the zero normal pressure gradient condition. While on the left and right sides: $\boldsymbol{U} \cdot \boldsymbol{n}=0$, where $\boldsymbol{n}$ is a unit vector perpendicular to the boundaries. The initial conditions are: $u=v=0, p=1.0$, $\rho=1.0$. In the Cartesian coordinate system, 200 grid points in the longitude direction and 40 grid points in the transverse direction are used. The grid space is set as 0.0005 and the time interval is 0.00005 , as shown in Figure $1 \mathrm{~b}$. The numerical technique used here is MAC's method discussed above. A proper grid should be one in which the coordinate lines become closely spaced as the surface is approached. For the sake of stability, the computational domain must include all of analysis domain. The present calculations were made on a uniform grid throughout the physical space. In combination with rectangular geometry existing in the physical plane, this means that no grid transformation is needed.

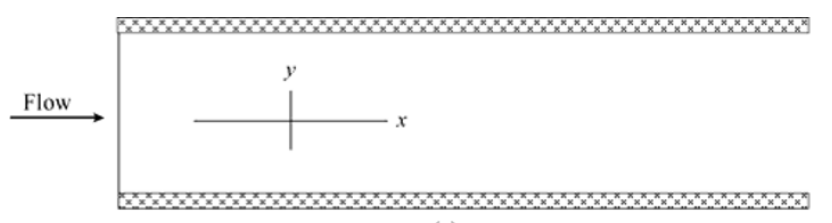

(a)

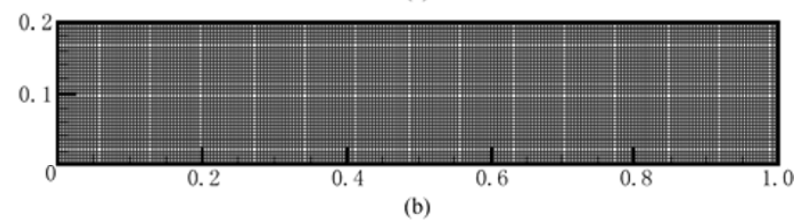

Figure 1 (a) Geometry and boundary conditions for flow through a single fracture; (b) Grids in the model for calculating $2 \mathrm{D}$ flow through a single fracture.

\section{Results and discussion}

In this section we shall demonstrate the accuracy and efficiency of the MAC method for incompressible flows on uniform grids. The geometry and boundary conditions are schematically shown in Figure 1a. Steady flow at a Reynolds number $R e=100$ is considered first. Calculations were performed on uniform grids with temporal step size of 0.0005 . The profile of horizontal velocity at $R e=100$ are shown in Figure 2a, from which one can observe that the MAC solution corresponds to the exact solution reasonably well. Effects of Reynolds number on velocity profiles are taken into account. Typical results obtained from the model mentioned above are given in Figure 2b. As anticipated, the increase of the Reynolds number reduces the drag force and hence causes the velocity to increase. The Reynolds number is the ratio of inertia to viscous forces. With high Reynolds number, the inertia forces dominate the physical phenomenon of a flow. Cardenas et al (2007) clearly demonstrated that inertial forces can significantly 

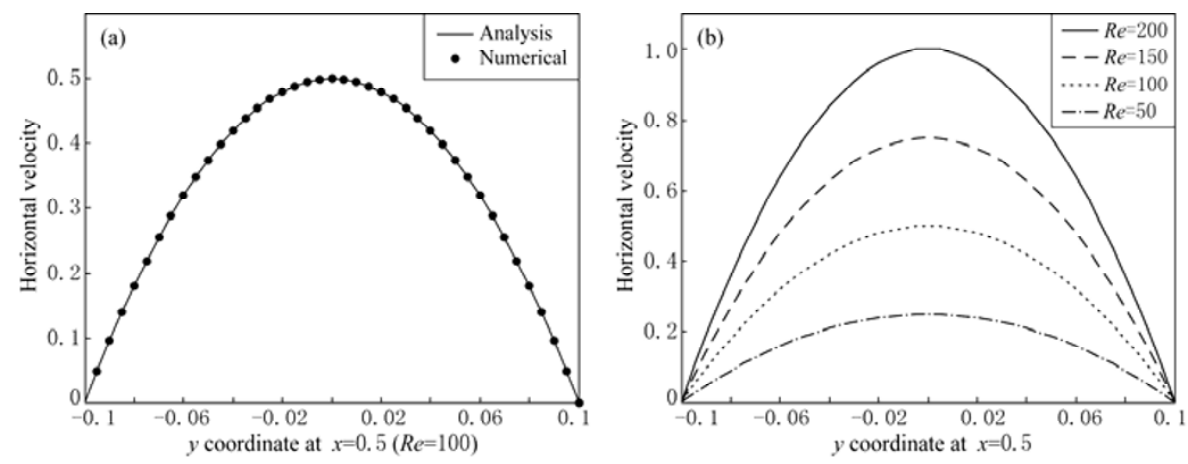

Figure 2 (a) Comparison of the numerical solution with the analytical solution with velocity profile at the horizontal centerline on a uniform grid at $R e=100$; (b) Velocity profile at the horizontal centerline as function of Reynolds number.

influence the internal flow field within a fracture and the total flow rate across a fracture. Figure 3 shows isobars (above) and velocity vectors (below) in a 2D flow through a single fracture at $R e=100$. We now look at the estimation of iteration divergence errors. First, an accurate solution was obtained by iterations until the residual norm became negligibly small (of the order of the round-off error in double precision). Then the calculation was repeated and the divergence error was computed as the difference between the converged solution obtained earlier and the intermediate solution. Figure 4 shows the norm of the divergence error. The divergence error is reduced with the increase of iterative times. The MAC scheme shows non-monotonic divergence towards the time step is independent of solution. Divergence error was reduced, as the time step size decreased. It can be observed from Figure 4 that, when the time step decreases, the peaks of the divergence error curves become smaller. The peak divergence error
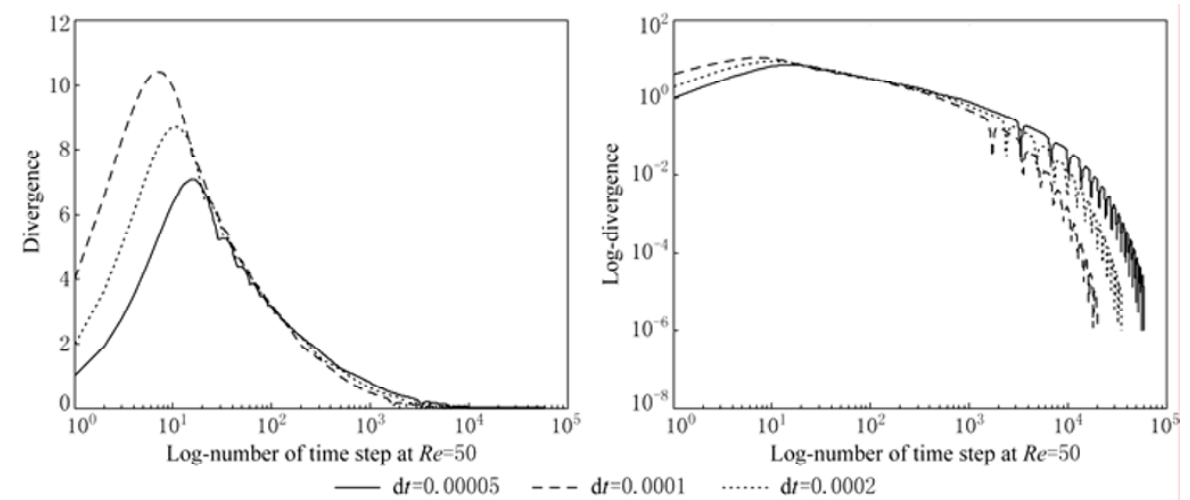

Figure 4 Effects of number of time step on the divergence.

In the next example, we study the impact of imposed pressure fluctuations on the flow dynamic behav- shifts along the time step axis depending on the time step size, suggesting that the peaks of these divergence error curves are sensitive to the time step. The phase of divergence error shifts towards the iteration number increasing direction. We denote that the divergence error is associated with equation (7).
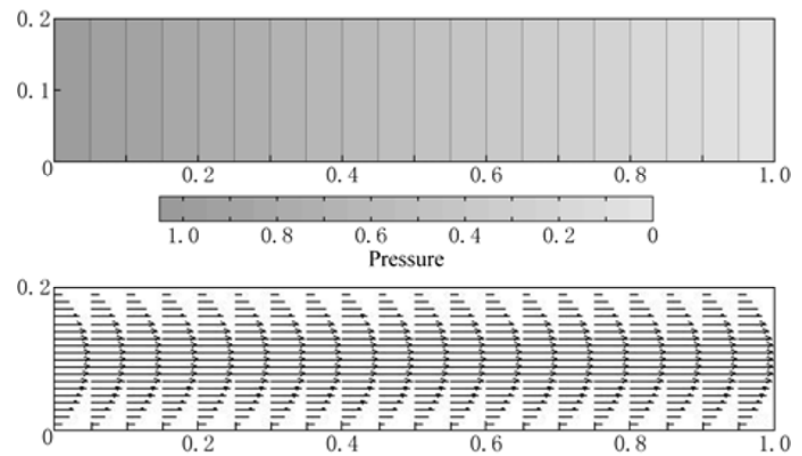

Figure 3 Isobars (above) and velocity vectors (below) in the 2D flow through a single fracture at $R e=100$. ior through a single fracture. Pressure fluctuations are important for predicting fluid flow through fractures. 
Because pressure is a major control of fluid flow, it would be enormously important to understand the dependence of fluid flow on pressure fluctuations. Assume the imposed pressure fluctuation is the following harmonic waveform:

$$
p=A \sin (\omega t)
$$

where $p$ is the pressure, $A$ is the amplitude, $\omega$ is the oscillating frequency and $t$ refers to the time. This expression for a plane-wave propagating in a single fracture is added on the left pressure boundary in Figure 1a. Now we analyze the effect of oscillating frequency on the flow behavior through a single fracture. In Figure 5, the different curves correspond to different frequencies between $10 \mathrm{~Hz}$ and $100 \mathrm{~Hz}$ at the Reynolds number of 50 . In general, the curves show an increase of horizontal velocity as the frequency reduces. For the range of frequency between $10 \mathrm{~Hz}$ and $50 \mathrm{~Hz}$, when the frequency increases, phases of the peaks of the velocity curves shift clearly, while the velocity peaks are almost same. It appears that for this range of low frequency, the pressure fluctuation mainly dominates the phase behavior of velocities.

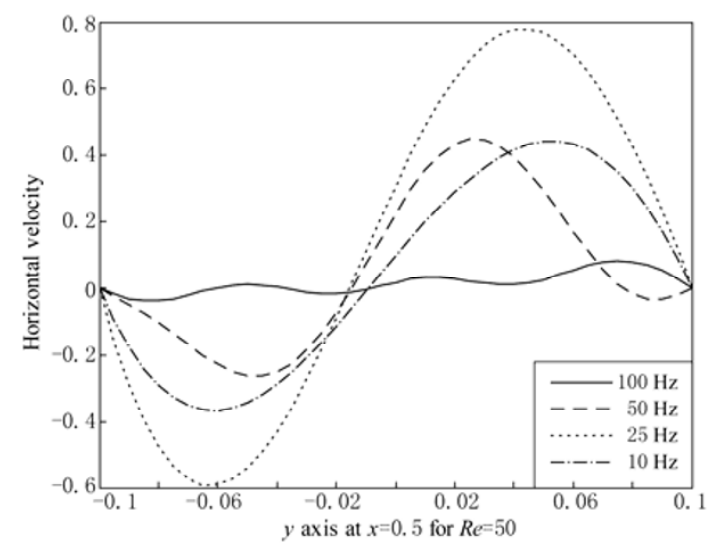

Figure 5 Effects of frequency on profiles of the horizontal velocity for Reynolds number of 50 .

The low-frequency limit of this pressure fluctuation corresponds to the flow phase governing fluid flow through a single fracture. As expected, the pressure fluctuations of the harmonic waveform have complex impact on the flow dynamical behavior. In Figure 5, for frequency of $100 \mathrm{~Hz}$, the effect of the pressure fluctuation becomes smaller compared with other frequencies. While for frequency of $25 \mathrm{~Hz}$, the pressure fluctuation has the maximum impact on the flow behavior. The low $(25 \mathrm{~Hz})$ - and high-frequency $(100 \mathrm{~Hz})$ results correspond to the upper and lower bounds of velocity induced by the pressure fluctuation. From this result, we can identify an effective frequency band (ranging from 10 $\mathrm{Hz}$ to $50 \mathrm{~Hz}$ ) of the pressure fluctuation which dominates dynamic behavior of the flow. We also note that there exits a critical frequency $(100 \mathrm{~Hz})$ of the pressure fluctuation which controls approximately the flow dynamic behavior. These results suggest that the effect of pressure fluctuation-induced fluid flow can be observed in a much broader frequency range. The peak velocity shifts along the spatial axis depending upon the frequency of the pressure fluctuation. The above analyses demonstrate that although there are some differences between the velocity amplitudes but solutions show the similar behavior for oscillating flow in a qualitative sense. Despite the fact that these results are based on the assumption of such special pressure fluctuation, it is shown that the essential effect of pressure fluctuations namely, a phase shift and amplitude variation of the pressure fluctuation-induced flow through a single fracture - can be modeled. It is inferred that estimation of flow behavior from pressure fluctuation is only possible if effective frequency range is properly accounted for.

\section{Conclusions}

The paper presents the numerical solution of fluid flow through a single fracture. With staggered marker-and-cell (MAC) and the Chorin pressure iteration finite difference method, the Navier-Stokes equation (NSE) solver was developed. It is stated that this NSE solver must obey the stability criterion embodied in equation (9) and it can be easily extended to three dimension. The equations for the conservation of mass and momentum were solved along with appropriate boundary conditions to determine the distribution of velocity components and pressure. Results were expressed in dimensionless form using Reynolds number. It was found that the flow is dominated by the relative importance of inertial and viscous effects. We identify an effective frequency band of the pressure fluctuation which dominates dynamic behavior of the flow. We also note that there exits a critical frequency of the pressure fluctuation which controls approximately the flow dynamic behavior. The effect of pressure fluctuation-induced fluid flow can be observed in a broader frequency range. The peak velocity shifts along the spatial axis depending upon the frequency of the pressure fluctuation. And more importantly, any attempt to estimate flow behavior from pressure fluctuation will fail, if the effective fre- 
quency range is not properly accounted for.

Acknowledgments This research is partly sponsored by National “973” Project (2006CB705802).

\section{References}

Brown S R (1995). Simple mathematical model of a rough fracture. $J$ Geophys Res 100: 5 941-5 952.

Brown S R, Stockman H W and Reeves S J (1995). Applicability of the Reynolds equation for modeling fluid flow between rough surfaces. Geophys Res Lett 22(18): 2 537-2 540.

Brush D J and Thomson N R (2003). Fluid flow in synthetic rough walled fractures: Navier-Stokes, Stokes, and local cubic law simulations. Water Resour Res 39(4): 1 085, doi:10.1029/2002WR001346.

Cardenas M B, Slottke D T, Ketcham R A and Sharp Jr J M (2007). Navier-Stokes flow and transport simulations using real fractures shows heavy tailing due to eddies. Geophys Res Lett 34: L14404, doi:10.1029/2007GL030545.

Giacomini A, Buzzi O, Ferrero A M, Migliazza M and Giani G P (2008). Numerical study of flow anisotropy within a single natural rock joint. International Journal of Rock Mechanics and Mining Sciences 45(1): 47-58.

Harlow F H (1965). Computer experiments in fluid dynamics. Scientific American Article 212(3): 104-110.

Jaramillo J E, Pérez-Segarra C D, Oliva A and Claramunt K (2007). Analysis of different RANS models applied to turbulent forced convection. Interna- tional Journal of Heat and Mass Transfer 50: 3 749-3 766.

Karpyn Z K, Grader A S and Halle P M (2007). Visualization of fluid occupancy in a rough fracture using micro-tomography. Journal of Colloid and Interface Science 307: 181-187.

Kim I, Lindquist W B and Durham W B (2003). Fracture flow simulation using a finite-difference lattice Boltzmann method. Phys Rev E 67: 046708.

Luis F, Ayala H, Ertekin T and Adewumi M (2007). Numerical analysis of multi-mechanistic flow effects in naturally fractured gas-condensate systems. Journal of Petroleum Science and Engineering 58: 13-29.

Li S, Dong M, Li Z, Huang S, Qing H and Nickel E (2005). Gas breakthrough pressure for hydrocarbon reservoir seal rocks: implications for the security of long-term $\mathrm{CO}_{2}$ storage in the Weyburn field. Geofluids 5: 326-334.

Michael J and Nicholl R L (2001). Simulation of flow and transport in a single fracture: Macroscopic effects of underestimating local head loss. Geophys Res Lett 28: 4 355-4 358.

Olivier H and Grönig H (1986). The random choice method applied to two-dimensional shock focusing and diffraction. Journal of Computational Physics 63: 85-106.

Steven H, Emerman D L, Turcotte and Spence D A (1986). Transport of magma and hydrothermal solutions by laminar and turbulent fluid fracture. Physics Earth Planet Inter 41: 249-259.

Waite M E, Ge S, Spetzler H and Bahr D B (1998). The effect of surface geometry on fracture permeability: A case study using a sinusoidal fracture. Geophys Res Lett 25: 813-816.

Zimmerman R W, Yaarubi A, Pain C C and Grattoni C A (2004). Non-linear regimes of fluid flow in rock fractures. International Journal of Rock Mechanics and Mining Sciences 41: 163-169. 